\title{
Reflection holograms in a PVA/AA photopolymer: Several compositions
}

\author{
Rosa Fuentes* Elena Fernández, Celia García and Inmaculada Pascual \\ Dpto. Óptica, Farmacología y Anatomía, Universidad de Alicante, Apartado 99, E-03080 \\ Alicante, Spain \\ *E-mail: fuentes@ua.es
}

\begin{abstract}
High spatial-frequency fringes associated with reflection HOEs are difficult to obtain with currently available recording materials. In this work holographic reflection gratings were stored in a PVA/acrylamide photopolymer. This material is formed of acrylamide photopolymer which is considered interesting material for optical storage applications such as holographic memories. The experimental procedure for examining the high spatial frequency response of this material is explained and the experimental results obtained are presented. With the aim of obtaining the best results, the performance of different material compositions is compared.
\end{abstract}

Keywords: Photopolymers, holographic recording materials, reflection holograms.

\section{INTRODUCTION}

Due to the importance acquired by new technologies (computers and Internet), the demand for storage techniques with more capacity, more density and faster readout rates has increased considerably. The conventional optical memory technologies, like CD-ROM's and DVD's, are two-dimensional surface-storage techniques, and so have almost arrived at the limit of their capacity. For this reason, in recent years a lot of attention has been centred on three-dimensional holographic disks ${ }^{1-3}$. Recently, many studies have focused on the characterization and optimization of thick holographic recording materials in order to obtain the maximum data storage capacity ${ }^{4}$.

Reflection holographic optical elements can be recorded for instance in silver halide sensitized gelatin emulsions ${ }^{5}$ with good results, but this recording medium has a thickness of $7 \mu \mathrm{m}$ which is very low if the aim is to store a great number of holograms in the same volume of material, as in the case of a holographic memory.

Since photopolymers have excellent holographic characteristics, such as high refractive index modulation ${ }^{6,7}$, large dynamic range ${ }^{2,8,9}$, good light sensitivity, real time image development, high optical quality and low cost, they have been used as the base of new 3-D holographic disks. In addition, their properties like energetic sensitivity or spectral sensitivity can be easily changed by modifying their composition ${ }^{6,8,10,11}$.

The photopolymer is composed of acrylamide (AA) as the polymerizable monomer, triethanolamine (TEA) as radical generator, N,N'-methylene-bis-acrylamide (BMA) as cross-linker, yellowish eosin (YE) as sensitizer and a binder of polyvinyl alcohol (PVA) ${ }^{6,10}$. The resulting layers are about $80 \pm 10 \mu \mathrm{m}$ thick.

Reflection holograms with an acrylamide-based photopolymer have been investigated for bit-format holographic data storage, and holographic reflection gratings have been recorded with a micrometer diameter and $0.28 \%$ diffraction efficiency ${ }^{12}$. This type of hologram has also been used for other applications such as a visual indication of environmental humidity ${ }^{13}$.

In this work, we focus on the optimization of the composition of a PVA-acrylamide photopolymer, as well as exposure time and intensity, with the aim of recording reflection holograms with a spatial frequency of up to $5000 \mathrm{lines} / \mathrm{mm}$ with the best results.

*fuentes@ua.es; phone +34 96590 9631; fax +34 965903746

Holography: Advances and Modern Trends, edited by Miroslav Miler, Miroslav Hrabovský

Proc. of SPIE Vol. 7358, 73581D · C 2009 SPIE · CCC code: 0277-786X/09/\$18 - doi: 10.1117/12.820628

Proc. of SPIE Vol. 7358 73581D-1 


\section{EXPERIMENTAL}

\subsection{Different compositions for recording material}

The holograms are registered in a photopolymer composed of acrylamide (AA) as the polymerizable monomer, triethanolamine (TEA) as radical generator, N,N'-methylene-bis-acrylamide (BMA) as cross-linker, yellowish eosin (YE) as sensitizer and a binder of polyvinyl alcohol (PVA). Adding BMA to the composition improves the energetic sensitivity and diffraction efficiency of the material and, in addition, gives a greater stability to the stored grating, thereby preventing it from disappearing with time.

A solution of PVA in water forms the matrix and this is used to prepare the mixture of AA, BMA, and photopolymerization initiator system composed of TEA and YE. The mixture is made under red light, deposited by gravity on a $22 \times 40 \mathrm{~cm}$ glass plate and left in the dark for $18-20$ hours to allow the water to evaporate in normal environmental conditions (temperature, $\mathrm{T} \approx 20^{\circ} \mathrm{C}$ and relative humidity, $\mathrm{RH} \approx 35 \%$ ). These conditions of drying time are optimized to obtain the maximum diffraction efficiency of the gratings. Once dry, the glass is cut into squares of $5 \times 5 \mathrm{~cm}$

Three different compositions were studied. Composition A has only acrylamide as a monomer, composition B also contains bisacrylamide as cross-linker, and composition C includes sodium formate, a chain transfer agent (CTA) ${ }^{14}$. Table 1 shows the component concentrations of the photopolymer compositions used to obtain layers about $80 \mu \mathrm{m}$ thick.

Table 1: Concentrations of the photopolymer compositions

\begin{tabular}{|c|c|c|c|}
\hline & Composition A & Composition B & Composition C \\
\hline Polyvinylalcohol & $8.26 \% \mathrm{w} / \mathrm{v}$ & $8.26 \% \mathrm{w} / \mathrm{v}$ & $8.26 \% \mathrm{w} / \mathrm{v}$ \\
Acrylamide & $0.44 \mathrm{M}$ & $0.44 \mathrm{M}$ & $0.44 \mathrm{M}$ \\
Triethanolamine & $0.2 \mathrm{M}$ & $0.2 \mathrm{M}$ & $0.2 \mathrm{M}$ \\
Yellowish Eosin & $2.4 \cdot 10^{-4} \mathrm{M}$ & $2.4 \cdot 10^{-4} \mathrm{M}$ & $2.4 \cdot 10^{-4} \mathrm{M}$ \\
N,N'-methylene-bis-acrylamide & ----- & $0.026 \mathrm{M}$ & ---- \\
Sodium Formate & ----- & ---- & $0.026 \mathrm{M}$ \\
\hline
\end{tabular}

\subsection{Mechanism of hologram recording}

A photoreduction reaction is produced when the material is illuminated with a light beam of $514 \mathrm{~nm}$. The dye is excited to the high-energy state and then reacts with the coinitiator TEA, which is the electron donor, to produce a dye radical anion and a triethanolamine radical cation. The dye radical is not usually reactive enough to initiate polymerization but the TEA radical will react with an AA molecule and polymerization may then occur. The reaction occurred in a PVA matrix and we consider that the PVA matrix is inert. When the material is exposed to an interference fringe pattern, more monomer is polymerized and the amount of polymer formed increases with exposure.

In some papers ${ }^{14}$ the spatial frequency response of an acrylamide polyvinylalcohol-based photopolymer is improved by adding a chain transfer agent (CTA), sodium formate. Chain transfer agents are added with the aim of reducing the length of the polymer chains formed. These agents have the ability to stop the growth of a chain. Thus the spatially periodic concentration distribution in the material formed by polymer chains can disperse more rapidly. This paper ${ }^{14}$ presents the results for transmission holograms analyzing a range of spatial frequencies from 500 to 2750 lines $/ \mathrm{mm}$. It is proposed that the addition of a CTA will decrease the average length of the polymer chains formed and hence localize the chain growth to the bright regions of the interference pattern. Thus, more but shorter polymer chains are expected to be formed. If a reduction in the non-local effect can be achieved, the potential improvements in high-spatial frequency material response will be significant. 
In this work, the influence of this component (CTA) on reflection holograms with a spatial frequency of up to 5000 lines/mm is studied and compared with other compositions that do not contain this component. We replaced the crosslinker (bisacrylamide) in composition B by the same concentration of chain transfer agent (sodium formate) to obtain composition $\mathrm{C}$.

\subsection{Holographic setup}

The basic setup used to record holographic reflection gratings is shown in Fig. 1. Two beams, each with an intensity of $20.3 \mathrm{~mW} / \mathrm{cm}^{2}$, from an Ar-ion laser overlap at a photosensitive plate, producing an appropriate interference pattern at the plane of the material due to the formation of polyacrylamide in the regions of constructive interference

Holographic reflection gratings were recorded using the output beam from the laser which was split into two beams and then spatially filtered, using a microscope objective lens and a pinhole, and collimated to yield a plane-wave source of light at $514 \mathrm{~nm}$. The diameter of these beams was $1.5 \mathrm{~cm}$. The two laser beams were spatially overlapped at the recording medium but reached the opposite sides of the holographic plate with symmetrical geometry. Both beams impinged at an angle $\alpha=45^{\circ}$ with respect to normal incidence. The grating pitch established by the experimental recording condition was $\Lambda=0.194 \mu \mathrm{m}$, applying the equation below (therefore the spatial frequency of the recorded reflection gratings was 5145 lines $/ \mathrm{mm}$ ) where $\lambda$ is the wavelength of the laser light used for the recording of a hologram, $\Lambda$ is the closest separation between the fringes of the interference pattern in the recording layer, $\mathrm{n}$ is the refractive index of the photopolymer and $\theta$ is the angle between the beams in the recording material.

$$
\Lambda=\frac{\lambda}{2 n \operatorname{sen}\left(\frac{\theta}{2}\right)}
$$

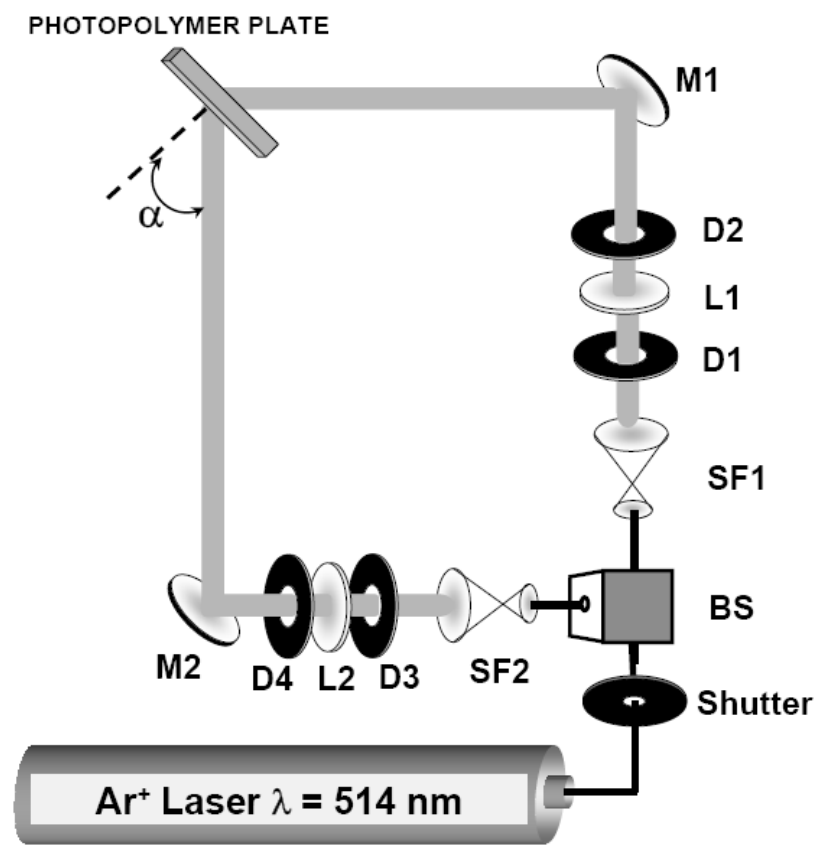

Figure 1: Experimental setup: BS beam splitter, Mi mirror, Li lens, Di, diaphragm, SFi, microscope objective lens and pinhole. 


\section{RESULTS}

The high spectral selectivity ${ }^{15}$ of the reflection holograms means that light of a specific wavelength is reflected when the hologram is illuminated with white light. The colour observed depends on the holographic fringe spacing $\Lambda$ and the reconstruction angle.

Each reflection grating was optically characterized by measuring its transmission spectrum. The dependence of transmittance on the wavelength was measured using a spectrophotometer. The transmittance values take into account Fresnel's reflections and the absorption of the glass substrate. The holographic gratings were then bleached by exposing them to a white light lamp to eliminate the dye and to avoid its absorption. The depth of the reflection peak on the transmittance curve allowed us to calculate the reflectance, that is, the diffraction efficiency of the recorded reflection grating. The angle of incidence at the reconstruction step was fixed at $0^{\circ}$, perpendicular to the plate (which is different to the Bragg angle at the recording stage) whereas the wavelength was now the free parameter. Therefore applying equation (1) with an angle $\theta$ of $180^{\circ}$, we obtain a theoretical value of $\lambda=582 \mathrm{~nm}$, at which the reflection peak should occur.

Fig 3, 4, 5 show the diffraction efficiency as a function of exposure, but these values do not represent the maximum diffraction efficiency values since the angle of incidence of the probe beam (normal incidence) of the spectrophotometer is different from that used during the recording $\left(45^{\circ}\right)$. The maximum diffraction efficiency obtained was $1.3 \%$ for composition A, $2.6 \%$ for composition B and $0.8 \%$ for composition C.

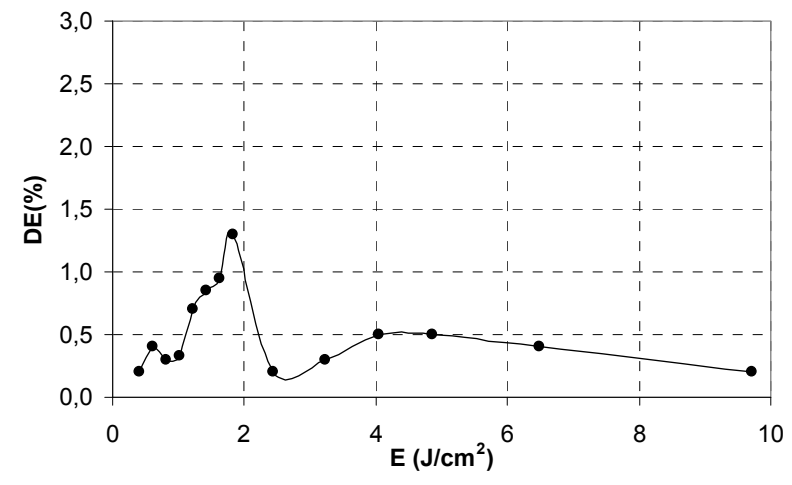

Fig.3: Diffraction efficiency as a function of exposure for reflection gratings recorded on plates with composition $\mathrm{A}$

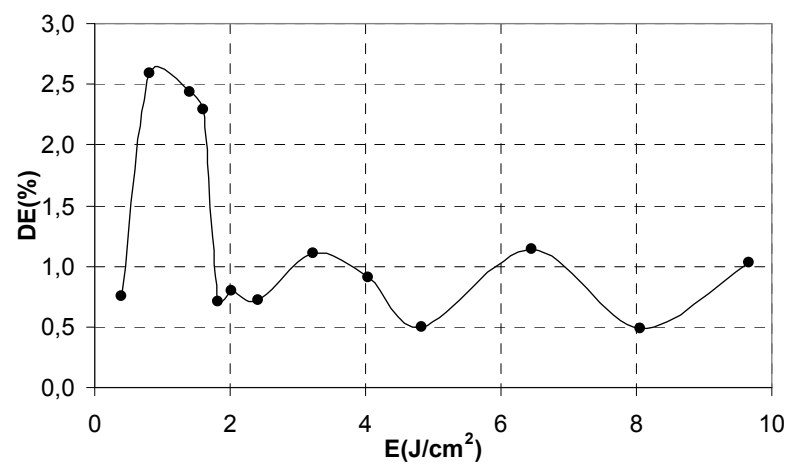

Fig.4: Diffraction efficiency as a function of exposure for reflection gratings recorded on plates with composition $\mathrm{B}$. 


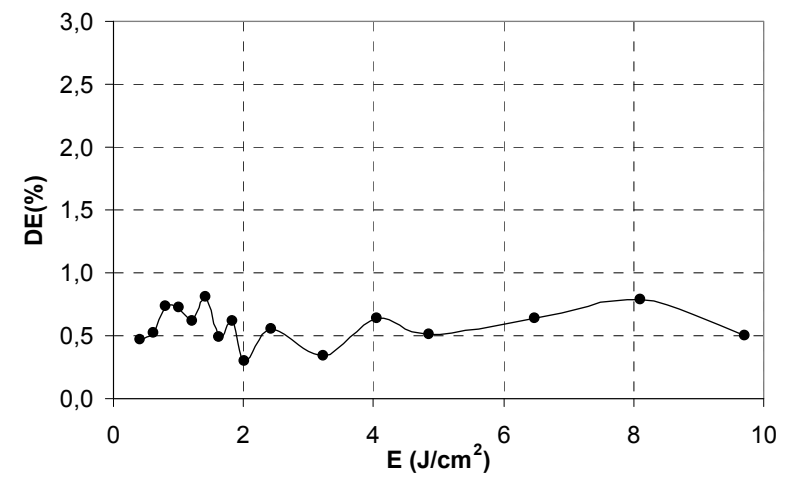

Fig.5: Diffraction efficiency as a function of exposure for reflection gratings recorded on plates with composition $\mathrm{C}$.

Fig. 6,7,8 show the transmittance of the plates with different compositions versus wavelength. They show the formation of the reflection peak and its wavelength shift.

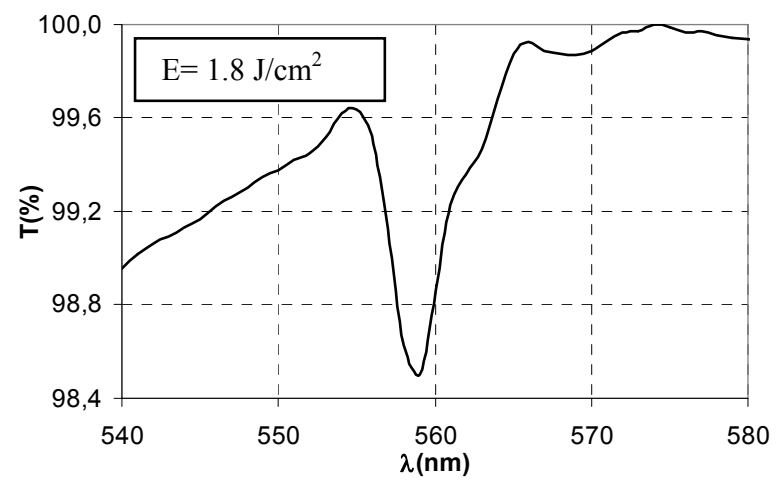

Fig.6: Transmittance as a function of the reconstruction wavelength for reflection gratings recorded on plates with composition A.

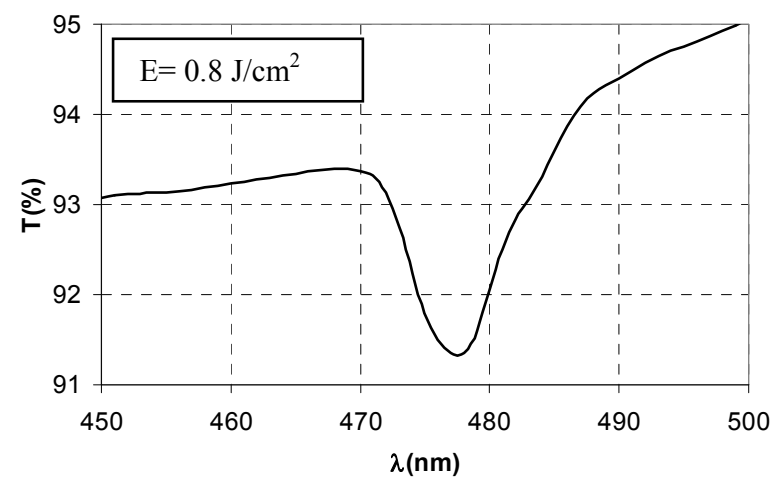

Fig.7: Transmittance as a function of the reconstruction wavelength for reflection gratings recorded on plates with composition B. 


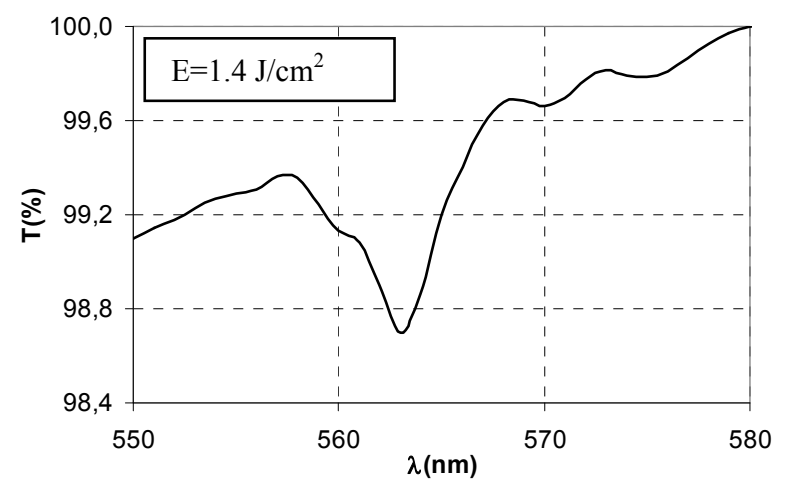

Fig.8: Transmittance as a function of the reconstruction wavelength for reflection gratings recorded on plates with composition C.

Removing the cross-linker from the material, as in composition A and C, has the effect of increasing the mobility of polyacrylamide chains, resulting in a decrease in the reflection diffraction efficiency.

The concentration of the chain transfer agent used $(0.026 \mathrm{M})$ is different from that used in other studies ${ }^{14}(0.007 \mathrm{M})$ and in our case, addition of a CTA was not seen to increase the diffraction efficiency when a high spatial frequency of up to 5000 lines $/ \mathrm{mm}$ is required. We believe that the next step will be to determine the concentration of this component necessary to improve the response of reflection holograms.

Dimensional changes in the recording medium can occur after recording and bleaching. Unfortunately during the photopolymerization process, the momonomer used usually undergoes volumetric changes; conversion of the monomer molecules into a polymer network is accompanied by close packing of the growing polymer chains and a subsequent reduction in volume. This phenomenon, known as photopolymerization shrinkage is particularly relevant in the case of reflection gratings whose pitch becomes lower than that defined by the geometrical conditions of the recording.

Shrinkage of the material induces a change in the fringe spacing $\Lambda$ in the thickness of the material. This results in a relevant shift of the wavelength at which the reflection peak appears. The ultimate effect is a change in the wavelength of the diffracted light with respect to the recording laser wavelength if the reconstruction takes place under Bragg's condition. However, in our case, the reconstruction was at normal incidence, which does not satisfy the Bragg condition. Therefore, our theoretical wavelength was considered as $\lambda_{\text {th }}=582 \mathrm{~nm}$, which is inferred by equation (1) and we calculated the final wavelength displacement with respect to this theoretical value.

It is possible to introduce ${ }^{16}$ a parameter called optical shrinkage as:

$$
s_{\text {opt }}=\frac{\lambda_{t h}-\lambda_{\text {exp }}}{\lambda_{t h}}
$$

This parameter represents the ratio between the final reflection wavelength displacement observed by spectral analysis $\left(\lambda_{\text {th }}-\lambda_{\text {exp }}\right)$ and the theoretical reflection wavelength of the grating if there was no shrinkage, and it was reconstructed not satisfying the Bragg condition $\lambda_{\text {th }}$

The behaviour of our samples shows a peak shift of $23 \mathrm{~nm}$ corresponding to $4 \%$ of optical shrinkage for composition A, $104 \mathrm{~nm}$ corresponding to $18 \%$ for composition $\mathrm{B}$ and $19 \mathrm{~nm}$ corresponding to $3 \%$ for composition $\mathrm{C}$. Therefore, it is observed that for the holograms recorded using composition $\mathrm{C}$, which included the CTA, the optical shrinkage is less than for the others and therefore the reflected wavelength is more similar to the theoretical one.

We present here our preliminary results and it can be seen that the maximum diffraction efficiency (2.6\%) for reflection gratings was obtained with composition B and the least shrinkage (3\%) with composition C. Experimentally, a range of concentrations of both the chain transfer agent and the cross-linker should be examined so as to better understand the 
process which takes place in our photopolymer material when holographic reflections gratings with high spatial frequency are recorded.

\section{CONCLUSIONS}

The capacity of acrylamide-based photopolymers of different compositions to record holographic reflection gratings has been investigated for optical storage applications. In this work, our preliminary results on recording and readout of reflection holograms are presented. The high spatial resolution of the photopolymer material is demonstrated by recording reflection gratings with a spatial frequency of 5145 lines $/ \mathrm{mm}$. Three different compositions of recording material were studied and the best results in terms of diffraction efficiency were obtained for the composition which included bisacrlylamide. In this case the gratings were characterized by a diffraction efficiency of $2.6 \%$. On the other hand, the holograms recorded using the composition which included the chain transfer agent had the least optical shrinkage and there was 3\% relative displacement of the reflected wavelength from the theoretically expected value.

\section{ACKNOWLEDGMENTS}

This work was supported by the "Ministerio de Ciencia e Innovación" Spain, under project FIS 2008-05856-C02-01 and by the "Generalitat Valenciana" Spain, under project GVPRE 2008/135.

\section{REFERENCES}

[1] $\mathrm{Pu}, \mathrm{A}$. and Psaltis, D., "High-density recording in photopolymer based holographic three-dimensional disks," Appl. Opt. 35, 2389-2398, (1996).

[2] Schnoes, M., Ihas, B., Hill, A., Dhar, L., Michaels, D., Setthachayanon, S., Schomberger, G., Wilson, W. L., "Holographic data storage media for practical systems," in Practical Holography XVII and Holographic Materials IX, Tung H.Jeong, ed., Proc SPIE 5005, 29-37, (2003).

[3] Mcleod, R. R., Daiber, A. J., McDonald, M. E., Robertson, T. L., Slagle, T., Sochava, S. L., Hesselink, L., "Microholographic multilayer optical disk data storage," Appl. Opt. 44, 3197-3207, (2005).

[4] Coufal, H., Psaltis, D., Sincerbox, G. T., Holographic Data Storage, Springer-Verlag, New Cork, (2000).

[5] Kim, J. M., Choi, B. S., Choi, Y. S., Bjelkhagen, H. I., Phillips, N. J., "Holographic optical elements recorded in silver halide sensitized gelatin emulsions. Part 2. Reflection holographic optical elements," Appl. Opt. 41, 1522-1533, (2002).

[6] Gallego, S., Ortuño, M., García, C., Neipp, C., Beléndez, A., Pascual, I., "High-efficiency volume holograms recording on acrylamide and N,N ' methylene-bis-acrylamide photopolymer with pulsed laser," J. Mod. Opt. 52, 1575-1584, (2005).

[7] Gallego, S., Ortuno, M., Neipp, C., Marquez, A., Belendez, A., Pascual, I., "Characterization of polyvinyl alcohol/acrylamide holographic memories with a first-harmonic diffusion model," Appl. Opt. 44, 6205-6210, (2005).

[8] Hsu, K. Y., Lin, S. H., Hsiao, Y. N., Whang, W. T., "Experimental characterization of phenanthrenequinone-doped poly(methyl methacrylate) photopolymer for volume holographic storage," Opt. Eng. 42, 1390-1396, (2003).

[9] Mok, F. H., Burr, G. W., Psaltis, D., "System metric for holographic memory systems," Opt. Lett. 21, 896-898, (1996).

[10] Ortuño, M., Gallego, S., García, C., Neipp, C., Beléndez, A., Pascual, I., "Optimization of a $1 \mathrm{~mm}$ thick PVA/acrylamide recording material to obtain holographic memories: method of preparation and holographic properties," Appl. Phys. B 76, 851-857, (2003). 
[11] Yao, H., Huang, M., Chen, Z., Hou, L., Gan, F., "Optimization of two-monomer-based photopolymer used for holographic recording," Mat. Lett. 56, 3-8, (2002).

[12] Jallapuram, R., Naydenova, I., Howard, R., Frohmann, S., Orlic, S., Eichler, H. J., "Acrylamide-based photopolymer for microholographic data storage," Opt. Mat. 28, 1329-1333, (2006).

[13] Naydenova, I., Jallapuram, R., Toal, V., Martin, S., "A visual indication of environmental humidity using a color changing hologram recorded in a self-developing photopolymer," Appl. Phys. Lett. 92, (2008).

[14] Gleeson, M. R., Sabol, D., Liu, S., Close, C. E., Kelly, J. V., Sheridan, J., "Improvement of the spatial frequency response of photopolymer materials by modifying polymer chain length," J. Opt. Soc. Am. B 25, 396-406, (2008).

[15] Hariharan, P., "Optical Holography Principles, Techniques and Applications", Cambridge University Press, New York, (1996).

[16] Criante, L., Beev, K., Lucchetta, D. E., Simoni, F., "Spectral analysis of shrinkage in holographic materials suitable for optical storage applications," in Holography 2005: International Conference on Holography, Optical Recording, and Processing of Information, Y. Denisyuk, V. Sainov, and E. Stoykova, eds., Proc. of SPIE 6252, 62520G-1-62520G-5, (2006). 